\title{
Title: Complete Atrioventricular Heart Block from an Epilepsy Treatment
}

\section{Shorter Running Title: AV Block as a Late Effect of Epilepsy Treatment}

\author{
Author names and affiliations: \\ Kelly M. Brinkley, MD $^{1}$ \\ ${ }^{1}$ Pediatrics-Neurodevelopmental Disabilities Residency Program, Indiana University School of \\ Medicine, 705 Riley Hospital Drive, Indianapolis, IN, 46202, USA. E-mail: keagan@iupui.edu.
}

Mark D. Ayers, $\mathrm{MD}^{2}$

${ }^{2}$ Department of Cardiology, Indiana University School of Medicine, 705 Riley Hospital Drive, RR 127, Indianapolis, IN, 46202, USA. E-mail: mdayers@iu.edu

Deborah K. Sokol, PhD, MD ${ }^{3}$

${ }^{3}$ Department of Neurology, Indiana University School of Medicine, 705 Riley Hospital Drive, Indianapolis, IN, 46202, USA. E-mail: dksokol@iupui.edu.

\section{Corresponding author:}

Kelly M. Brinkley, MD, Pediatrics-Neurodevelopmental Disabilities Residency Program, Indiana University School of Medicine, 705 Riley Hospital Drive, Indianapolis, IN, 46202. Email: keagan@iupui.edu.

Word count: 451 words (inclusive of references and figure caption)

This is the author's manuscript of the article published in final edited form as:

Brinkley, K. M., Ayers, M. D., \& Sokol, D. K. (2017). Complete Atrioventricular Heart Block From an Epilepsy Treatment. Pediatric Neurology. https://doi.org/10.1016/j.pediatrneurol.2017.11.014 
Keywords: complete atrioventricular heart block; third degree heart block; vagus nerve stimulator; vagus nerve stimulation 
Atrioventricular (AV) heart block without adequate escape rhythm can result in sudden cardiac arrest and death. We report complete (third degree) AV block in a 16 year-old boy as a late effect of vagus nerve stimulation (VNS). He experienced brief, daily, complex partial seizures, treated with lamotrigine, levetiracetam, and the placement of Model 102 VNS at age 4.

Electroencephalography (EEG) showed generalized slow spike-and-wave discharges consistent with Lennox-Gastaut syndrome. At age 12, his VNS was changed to Model 303

PereniaDURA/Model 103 Demipulse generator, set at an output current of $2.25 \mathrm{~mA}$ on a standard 30 seconds on, 5 minutes off paradigm. At age 16, he experienced episodes of sudden collapse followed by unconsciousness. Holter monitor showed 15 second symptomatic complete AV block without escape (Figure 1). The patient was taking psychotropic medication with potential cardiac side effects: methylphenidate (tachycardia), guanfacine (first degree AV block), and haloperidol (prolonged QT interval, Torsades de Pointes). Haloperidol, methylphenidate, and VNS were stopped without further symptoms. Off these medications, the VNS was resumed at reduced current (1.25 mA), with re-occurrence of symptomatic AV block. Again, the VNS was stopped and symptoms/heart block ceased. Psychotropic medication was resumed without any side effects.

The vagus nerve innervates the heart. Therefore, precautionary practices include: (1) implanting the vagus nerve stimulator on the left side because the right vagus nerve innervates the sinoatrial node and its stimulation is more likely to cause bradycardia, asystole, or other adverse cardiac side effects, compared to the left vagus nerve which supplies the atrioventricular node ${ }^{1}$; and (2) arrhythmia tests performed during implantation. Still, late onset cardiac side effects occur, such as bradycardia ${ }^{2}$ and bradyarrythmia ${ }^{3}$ in children, and asystole in an adult ${ }^{4}$. In all cases, VNS was stopped, resulting in symptom relief and return to normal rhythm.

\section{Declaration of Conflicting Interests}

The authors declared no potential conflicts of interest with respect to the research, authorship, and/or publication of this article.

\section{Funding}

The authors received no financial support for the authorship and/or publication of this article.

\section{References}

[1] Randall WC, Ardell JL, Becker DM. Differential responses accompanying sequential stimulation and ablation of vagal branches to dog heart. Am J Physiol 1985; 249: 133-140.

[2] Cantarin-Extremera,V. et.al (2016). Late-onset periodic bradycardia during Vagus Nerve Stimulation in a pediatric patient. A new case and review of the literature. EJPN, 2016; 20: DOI:10.1016/j.ejpn.2016.02.014.

[3] Amark, P, et al (2007). Late onset bradyarrthmia during vagus nerve stimulation, Epilepsia 48: 1023-1024. DOI:10111/j.1528-1167.2007.01023x.

[4] Iriarte, J. et al (2008). Late-onset periodic asystolia during vagus nerve stimulation. Epilepsia 50:928-932. DOI:10.1111/j.1528-1167.2008.01918.x. 
Figure I. Complete AV block as a late effect of vagus nerve stimulation (VNS). This tracing, which is a continuous 24 second record, shows a 13 second pause with only p waves identifiable (arrow). 